\title{
DEVELOPMENT OF RETURN TO BASE FLIGHT TRAJECTORY GENERATOR BASED ON DUBINS PATH - VECTOR FIELD METHOD
}

\author{
Neno Ruseno ${ }^{(1) *}$, Muhammad Royyan(1), Prianggada I. Tanaya(2) \\ (1) Department of Aviation Engineering, International University Liaison Indonesia \\ (2) Department of Industrial Engineering, International University Liaison Indonesia \\ ${ }^{*}$ Corresponding Author: neno.ruseno@iuli.ac.id
}

\begin{abstract}
In a Return to Base (RTB) situation, aircraft needs to immediately fly back to its origin airport. Since there is no published flight procedure for an RTB, an Air Traffic Controller (ATC) will assist the pilot for the flight procedure to fly. The objective of this work is to generate a flight trajectory in RTB situation based on the available airport flight procedures (departure and arrival) in Kertajati airport. The Dubins Path was used as a method to generate the flight trajectory and supported by the Vector-Field Methodology. The Python programming simulation was used to simulate the flight trajectory generation in the normal condition, second closest waypoint condition, and different parameters value condition. The trajectory was simulated based on flight characteristic of a single engine aircraft (Cesna 172) and multi-engine aircrafts (Boeing 737-800NG). The simulation results show that the Dubins Path and Vector-Field methodology success to generate the flight trajectory in different types of condition and parameters. The increase in aircraft velocity and the decrease in aircraft bank angle caused an increase in the aircraft turning radius. While, the decrease in aircraft flight path angle caused increase in the length of Dubins Path line.
\end{abstract}

Keywords: Dubins Path, Flight Trajectory Generation, Return to Base, Vector Field Methodology

\section{INTRODUCTION}

Return to Base is an emergency situation when an aircraft need to return to their origin airport. There are two causes that make an RTB occurred, a technical problem and a non-technical problem. The aircraft will be required to land as soon as possible to avoid further problem or even the aircraft crash to the ground. Since there is no published flight procedure for an RTB, an Air Traffic Controller (ATC) will assist the pilot for the flight procedure to fly.

In order to ease the ATC workload, a flight trajectory generation should be able to generate the most flyable trajectory that connects the current aircraft location to the arrival procedure.

Then the aircraft can continue to follow the published procedure for landing. In this paper, the focus of work is to generate a flight trajectory when an RTB occur.

One of the popular methods for trajectory generation is the Dubins
Path method. It was first introduced by Lester Eli Dubins [1] in 1957. Dubins Path is the shortest path that connects between two given configurations. There are several algorithms that assist the Dubins Path to generate a flight trajectory. First, the work conducted by Hota and Ghose [2] on an optimal path planning for an aerial vehicle in 3D space. In this work, the Dubins Path succeed to generate path planning using geometrical and numerical method. The geometrical method used to generate the real time trajectory solution. While the numerical method used to adjust the flight path angle so the Circle Straight Circle (CSC) path can be flyable based on a Multiple Shooting (MS). Second work on Dubins Path using the Genetic Algorithm (GA) was conducted by $Y u$ and Hung [3]. It show that the GA able to produce the possible solution and to revise it until the optimal solution was found. The GA method was able to produce a good Dubins Path result for both low and high waypoint density 
cases. However, the GA method became inefficient if the number of waypoints become very large. Other work conducted by Lin and Saripalli [4] concluded that the Dubins Path succeed to generate the flight trajectory by using the Rapidly exploring Random Tree (RRT). It was able to find the shortest path and the loop of execution to predict the obstacle motion.

In the study of Owen, Beard, and McLain [5], the aircraft Dubins Path was implemented on a fixed-wing UAV. It demonstrated ability to generate a flight trajectory in the low, medium, and high altitude differences. The low altitude difference trajectory had a normal path, whereas the medium altitude difference needed to add an additional immediate arc at the beginning or at the end part. For the high altitude, the trajectory needed to extend the path is by adding a certain number of spirals. In this work the Dubins Path method was combined with the Vector-Field algorithm. In another work, the Dubins Path was assisted by Lyapunov strategy to perform a simulation by finding a missing person by using a fixed-wing UAV. This work was proposed by Lugo-Cardanes, Flores, Salazar, and Lozano [6]. Paul, Hole, Zytek, and Varela [7] demonstrated the Dubins Path can also be used to generate a trajectory in an emergency situation. It combined the Trajectory Planning Algorithm and a new Dynamic Data Driven Avionics Software (DDDAS) approach to determine the aircraft actual capabilities and generate the possible landing trajectories.

For this work, we applied the Vector-Field Methodology to assist the Dubins Path to generate a flight trajectory on an aircraft in RTB situation. The Vector-Field methodology works by using the halfplane concepts which is divided by start half-plane, straight line, and final half-plane. The method was applied to the low altitude difference case for calculation of the Dubins Path length and flight path angle. The simulation of the flight trajectory generation was conducted in normal condition, second closest waypoint condition, and different parameters value condition.

The following paragraph is consisted of the Dubins Path description, Methodology used, showing the results, discussion on the finding, and the conclusions.

\section{DUBINS PATH ALGORITHM}

It starts with a simple case of 2 dimension trajectory and the requirements of Dubins Path. It continues with the Dubins Path for aircraft trajectory in 3 dimensions.

\subsection{Dubins Car Path}

Dubins Car path is the robotic model used to find the minimum distance between two configurations. The Dubins Car path configuration can be described by $(x, y, \theta)$. Where, the $(x, y)$ is the position at the plane and $(\theta)$ is the direction of the car. Therefore, the kinematic model of Dubins Car is:

$$
\begin{gathered}
\dot{x}=V \cos \theta \\
\dot{y}=V \sin \theta \\
\dot{\theta}=u
\end{gathered}
$$

Where (V) is the velocity, $(\theta)$ is orientation angle and $(\mathrm{u})$ is the control input [3].

It shows that the car model basically has only 3 controls to operate. The 3 controls are turn left at maximum (L), turn right at maximum $(\mathrm{R})$, and go straight (S). The combination of these controls will be used to create the Dubins paths. These controls can be classified as two classes: ' $S$ ' in order to go straight and ' $\mathrm{C}$ ' in order to turn both right and left. The shortest path combination is RSR, LSL, RSL, LSR, RLR, and LRL. From these combinations, the controls class are CSC and CCC [8]. 
To find the Dubins Path, the flight trajectory generation must know what are the initial configuration $\left(P_{i}\right)$, final configuration $\left(P_{f}\right)$, orientation angles: $\alpha$ and $\beta$. Those are used to determine their direction of motion. The orientation angles are divided into four quadrants as shown in Figure 1.

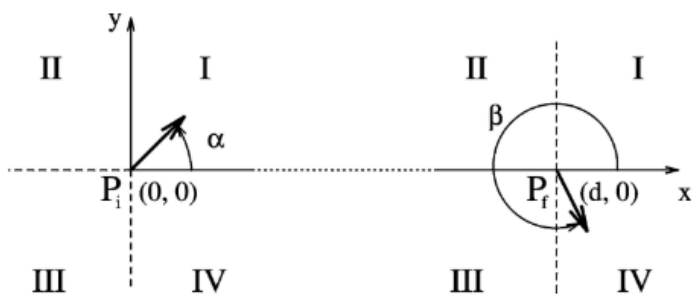

Figure 1: The Dubins Path Orientation Angles [9]

\subsection{Dubins Path Requirement}

In order to perform the Dubins Path calculation, the distance between start and final configurations must fulfill the Dubins Path requirement. The Dubins Path requirement is calculated based on the equation:

$$
D=\sqrt{\left(x_{2}-x_{1}\right)^{2}+\left(y_{2}-y_{1}\right)^{2}}
$$

Where $(D)$ is the distance between the center of initial Dubins circle and the center of final Dubins circle. The distance between start and final configurations must exceed the $D$ value as shown in Figure 2 [8].

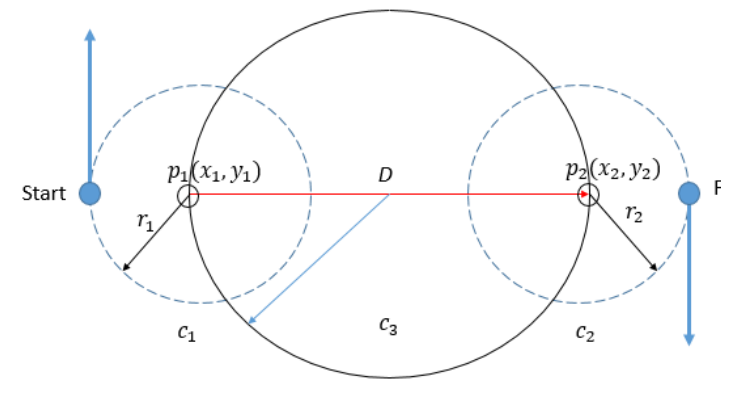

Figure 2. The Dubins Path Requirement

\subsection{Dubins Airplane Path}

The Dubins Airplane path is an extension of the Dubins Car path model. It is used to perform the threedimensional space simulation of an airplane. The differences between the Dubins Car and Dubins Airplane are the Dubins Airplane considers the altitude difference between start and final configuration, the Dubins Car path length, and the limit of flight path angle $(\gamma)$. Because of the altitude difference, the Dubins Airplane configuration consists of four variables: longitude, latitude, altitude, and heading angle $(x, y, z, \theta)$. Therefore, the kinematic model of the Dubins Airplane is as follow:

$$
\begin{gathered}
\dot{x}=V \cos \psi \cos \gamma \\
\dot{y}=V \sin \psi \cos \gamma \\
\dot{z}=-V \sin \gamma \\
\dot{\psi}=\frac{g}{V} \tan \phi
\end{gathered}
$$

where $(V)$ is the aircraft velocity, $(\psi)$ is the heading angle, $(g)$ is the gravity and $(\phi)$ is the bank angle [5].

The minimum turning radius for Dubins Airplane Path is,

$$
R_{\text {min }}=\frac{V^{2}}{g} \tan \phi
$$

In the Dubins Airplane Path, the path usually is defined by three cases: low altitude, medium altitude, and high altitude. In this work, it is assumed that the RTB situation only has a low altitude difference. Thus, the flight trajectory generation will produce the Dubins Airplane Path which consist only curve-straight-curve.

The low altitude case requirement is the altitude gain between the start and final configurations need to be,

$$
\Delta \text { altitude } \leq L_{i} * \tan \gamma
$$

Where the altitude gain can be obtain by flying at flight-path angle $\pm(\gamma)$ for a distance of $\left(L_{i}\right)$. If the requirement is fulfilled, the flight-path angle will be adjusted by,

$$
\gamma^{*}=\tan ^{-1} \frac{\Delta \text { altitude }}{L_{i}}
$$


After Dubins Path adjusted the flightpath angle, the length of Dubins Airplane Path is calculated by,

$$
L_{f}=\frac{L_{i}}{\cos \gamma^{*}}
$$

\section{METHODOLOGY}

In this work, some data such as procedure waypoints and aircraft parameters are required. The departure/arrival procedure waypoints were extracted from Aeronautical Information Publication (AIP) of
Kertajati airport. While, the aircraft parameters used in the simulation are Boeing 737-800NG and Cessna 172 Skyhawk. This section also covers Vector Field Methodology to support Dubins Path algorithm.

\subsection{Procedure waypoints}

From the AIP of Kertajati airport, the data extracted are runway heading, departure and arrival procedures [10]. The map of departure and arrival waypoints is shown in Figure 3 and the waypoint data is shown in Table 1.

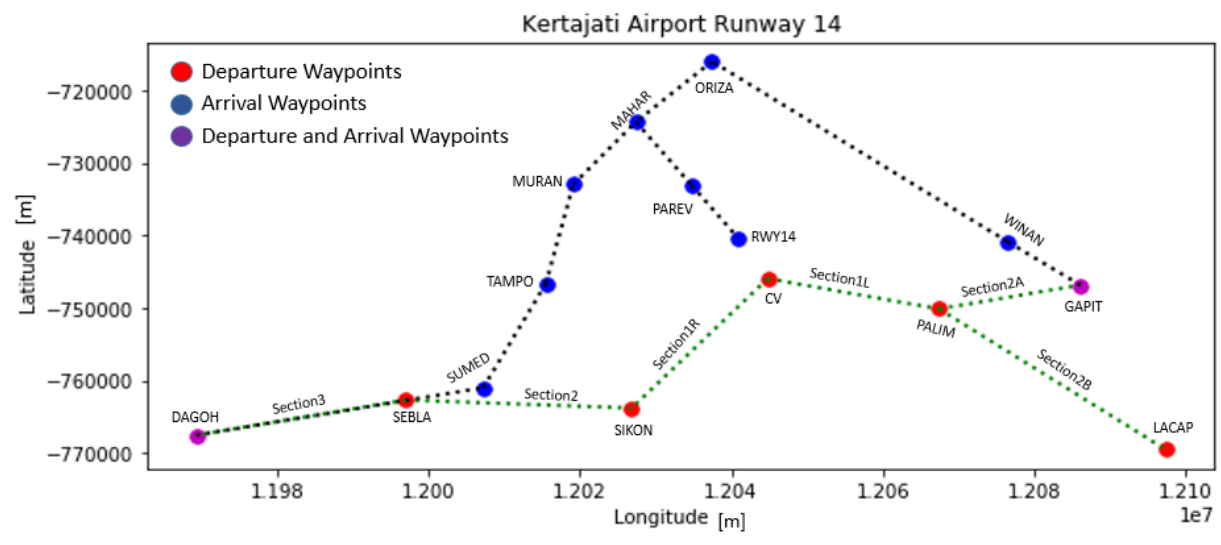

Figure 3. Aeronautical Information Publication

Table 1. Kertajati Flight Procedure Waypoints

\begin{tabular}{|c|c|c|c|c|c|c|c|c|c|}
\hline \multicolumn{5}{|c|}{ Departure RWY14 } & \multicolumn{5}{|c|}{ Arrival RWY14 } \\
\hline Waypoint & Longitude & Latitude & $\begin{array}{l}\text { Altitude } \\
(\mathrm{ft})\end{array}$ & Heading & Waypoint & Longitude & Latitude & $\begin{array}{l}\text { Altitude } \\
(\mathrm{ft})\end{array}$ & Heading \\
\hline CV & 108.195 & -6.69 & 1000 & $340^{\circ} / 225^{\circ}$ & GAPIT & 108.56 & -6.698 & 14500 & $149^{\circ}$ \\
\hline PALIM & 108.395 & -6.727 & 9000 & $10^{\circ} / 328^{\circ}$ & WINAN & 108.475 & -6.645 & 14500 & $149^{\circ}$ \\
\hline GAPIT & 108.56 & -6.698 & $\begin{array}{l}9000 \\
<x< \\
13000\end{array}$ & $10^{\circ}$ & ORIZA & 108.13 & -6.425 & 5000 & $220^{\circ}$ \\
\hline LACAP & 108.66 & -6.899 & $\begin{array}{l}x<= \\
13500\end{array}$ & $328^{\circ}$ & MAHAR & 108.041 & -6.499 & 3000 & $319^{\circ}$ \\
\hline SIKON & 108.036 & -6.848 & 8000 & $179^{\circ}$ & DAGOH & 107.512 & -6.881 & 11000 & $10^{\circ}$ \\
\hline SEBLA & 107.757 & -6.838 & 11000 & $190^{\circ}$ & SUMED & 107.847 & -6.823 & 11000 & $60^{\circ}$ \\
\hline DAGOH & 107.512 & -6.881 & 14000 & $190^{\circ}$ & TAMPO & 107.92 & -6.696 & 7000 & $75^{\circ}$ \\
\hline & & & & & MURAN & 107.952 & -6.575 & 5000 & $30^{\circ}$ \\
\hline & & & & & PAREV & 108.106 & -6.576 & 1700 & $319^{\circ}$ \\
\hline & & & & & RWY14 & 108.16 & -6.64 & 0 & $319^{\circ}$ \\
\hline
\end{tabular}

Table 2. Aircraft Performance Data

\begin{tabular}{|c|c|c|c|}
\hline \multicolumn{4}{|c|}{ Performance Data } \\
\hline \multicolumn{2}{|c|}{ Boeing 737-800 NG } & \multicolumn{2}{|c|}{ Cessna 172 Skyhawk } \\
\hline \multicolumn{2}{|c|}{ Climb Speed } & \multicolumn{2}{|c|}{ Climb Speed } \\
\hline IAS & 290 knots & IAS & 80 knots \\
\hline
\end{tabular}




\begin{tabular}{|l|c|l|c|}
\hline & $149.189 \mathrm{~m} / \mathrm{s}$ & & $41.156 \mathrm{~s}$ \\
\hline
\end{tabular}

\subsection{Aircraft Specifications}

The aircraft specification is the performance data of the aircraft to be implemented in the flight trajectory planning simulation. In this work, the flight trajectory generation used a single engine and a multi engine aircraft. The performance data consists of indicated airspeed for the maximum climbing speed as shown in Table 2.

\subsection{Vector-Field Methodology}

Vector-Field Methodology consists of switching between orbits and straight line (Figure 4). Based on the initial configuration $\left(\theta_{S}\right)$, the airplane will follow the center $\left(c_{s}\right)$ and direction $\left(\lambda_{s}\right)$ of orbit 1 . The orbit 1 will follow until it crosses the half-plane $H_{s}\left(w_{s}, q_{s}\right)$ or final turning radius. $w_{s}$ is a position on the half-plane and $q_{s}$ is unit vector orthogonal to the half-plane. The airplane will follow the straight line defined by $\left(w_{s}, q_{s}\right)$ until it arrive at halfplane $H_{l}\left(w_{l}, q_{l}\right)$.

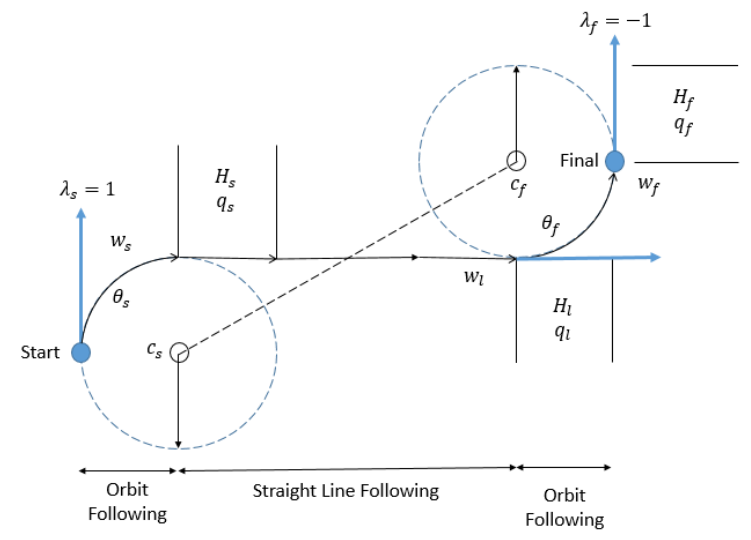

Figure 4. Vector-Field Methodology Dubins Path
The next step is similar to the start circle but with the difference in the final configuration. The description of Dubins Path is shown in Figure 3.2 [5].

\section{RESULTS AND DISCUSSION}

The simulation was performed in three categories, which were the simulation in normal condition, simulation in second closest waypoint condition, and simulation with different bank angle and flight path angle values. The first category used both aircrafts, but the second and third simulations only used Boeing 737-800NG (B738).

\subsection{Normal Condition}

The normal condition simulation was when the Dubins Path performed a simulation in the maximum bank angle $\left(25^{\circ}\right)$ and the maximum flight path angle $\left(45^{\circ}\right)$. The simulation performed in the left side of departure route. The aircraft position started at the 108.292 East longitude, -6.708 South latitude, 1487.655-meter altitude, and the direction of $340^{\circ}$ orientation angle. After Dubins Path calculated the initial waypoint, the flight trajectory generation obtained the WINAN arrival waypoint as the shortest distance to final waypoint with 21904.329 meter (Figure 5). The WINAN arrival waypoint had a position in the 108.475 East longitude, -6.645 South latitude, 4419.6-meter altitude, and $149^{\circ}$ orientation angle. The result of simulation is shown in Figure 5 to 6 for Boeing 737 and Figure 7 to 8 for Cesna 172. 


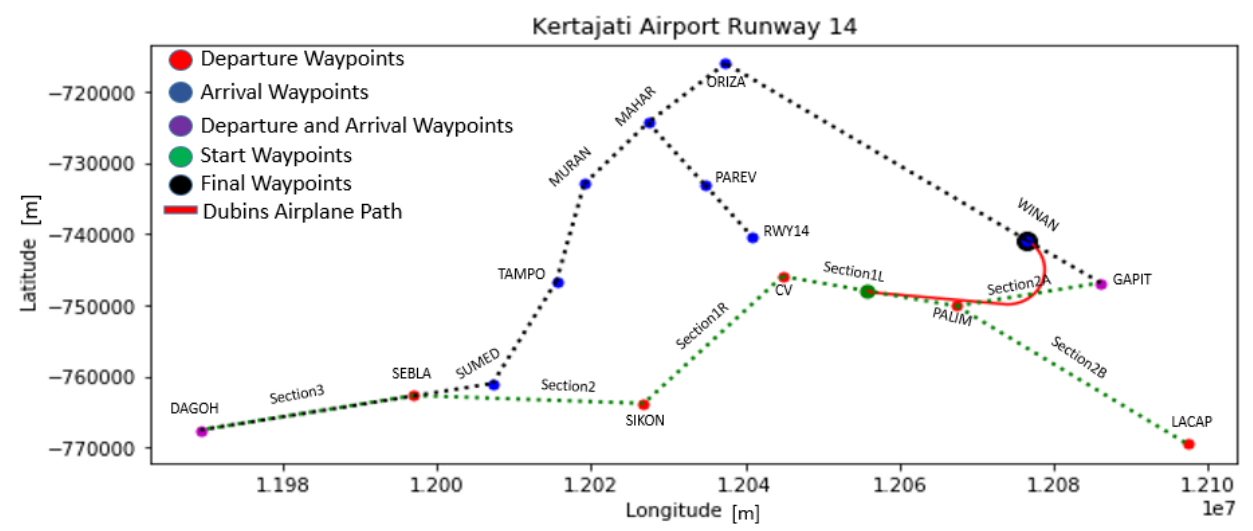

Figure 5. B738 Flight Trajectory Normal Condition (planview)
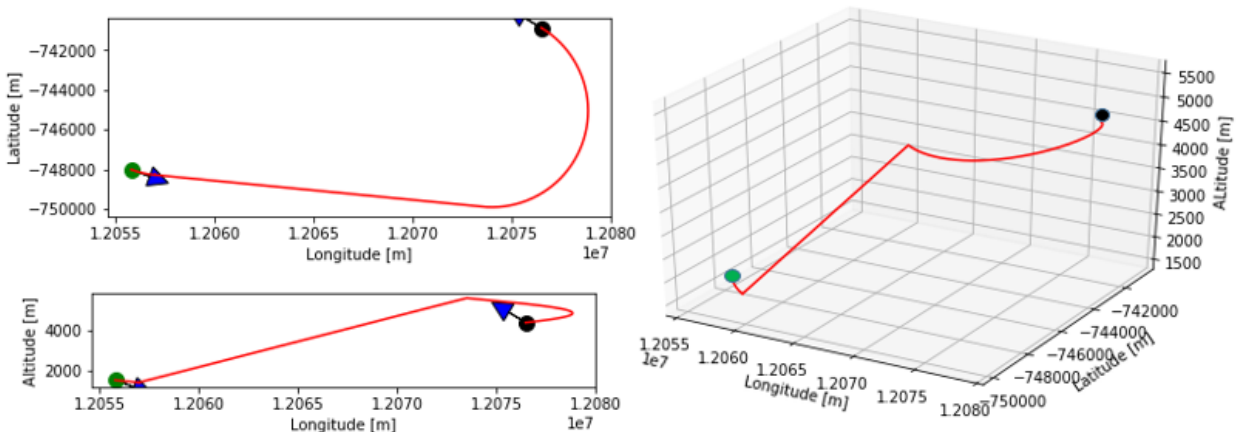

Figure 6. B738 Dubins Airplane Path in Normal Condition (2D and 3D)

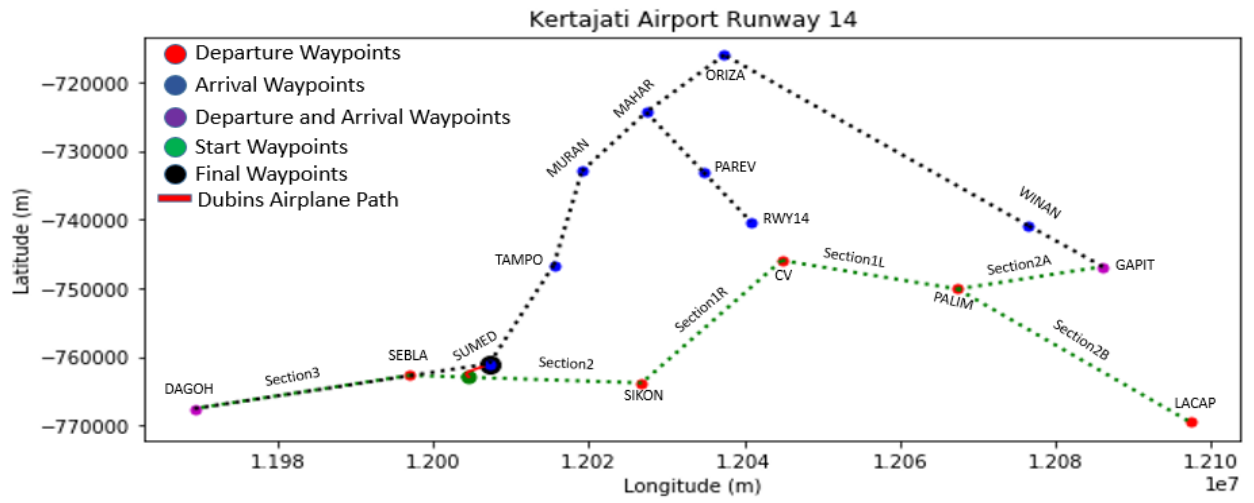

Figure 7. Cessna-172 Flight Trajectory in Normal Condition (planview)
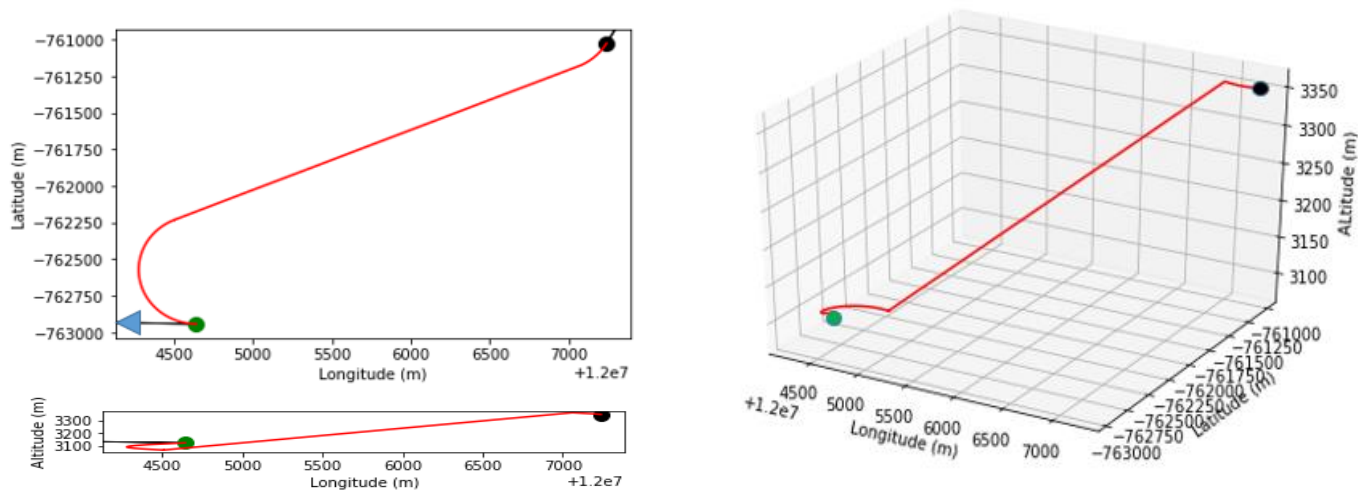

Figure 8: Cesna-172 Dubins Airplane Path in Normal Condition (2D and 3D) 
In the normal condition, the aircraft velocity has significant impact to the Dubins Airplane Path because the aircraft velocity determines the value of aircraft turn radius as shown in Equation 4. It shows that the B738 velocity $(149.189 \mathrm{~m} / \mathrm{s})$ cause the Dubins turn radius larger than the Cessna 172 Skyhawk (C172) turn radius.

\subsection{Second Closest Waypoint Condition}

The second closest waypoint condition was the condition where the aircraft position and the closest arrival waypoint were not meet the requirement to generate the Dubins Airplane Path. The simulation used the second shortest distance between start waypoint and final arrival waypoint and generated the Dubins Airplane Path.

The simulation was performed on the right side of departure route. The aircraft position started at the 107.522 East longitude, -6.879 South latitude, 4231.628-meter altitude, and $190^{\circ}$ orientation angle. The Dubins Path obtained the DAGOH arrival waypoint as the shortest distance to final waypoint with 7367.745 meter. After the flight trajectory generation checked the Dubins Airplane requirement, it turned out that it did not meet the requirement. The flight trajectory generation calculated the final arrival waypoint again with the second shortest arrival waypoint. The result showed that the SUMED arrival waypoint was the second shortest distance to final waypoint with 37285.732 meter (Figure 9). The SUMED arrival waypoint had a position in the 107.847 East longitude, -6.823 South latitude, 3352.8-meter altitude, and $60^{\circ}$ orientation angle.

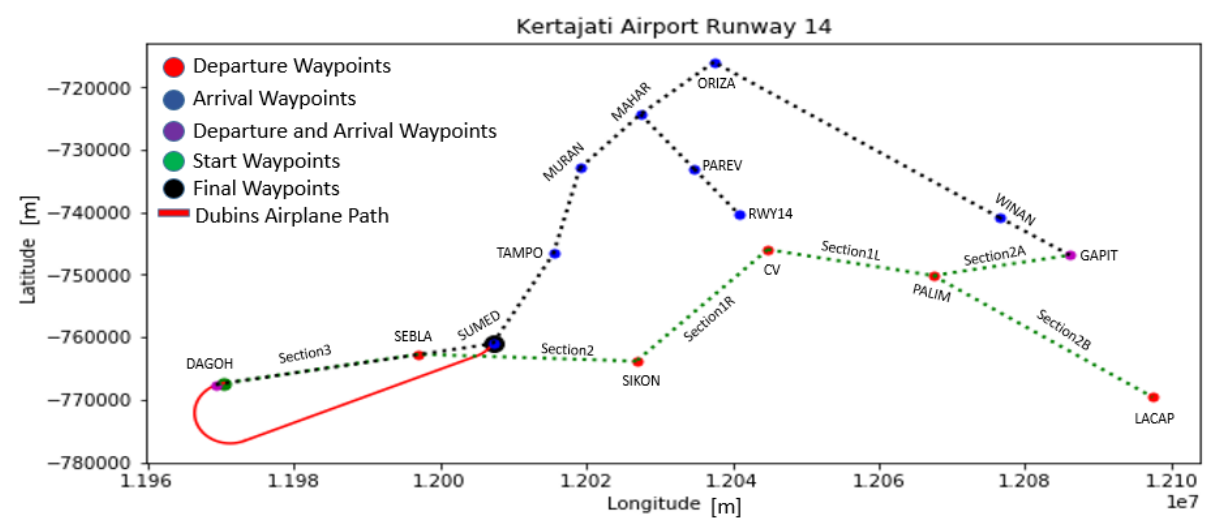

Figure 9. B738 Flight Trajectory in Second Closest Waypoint Condition on the Kertajati Airport
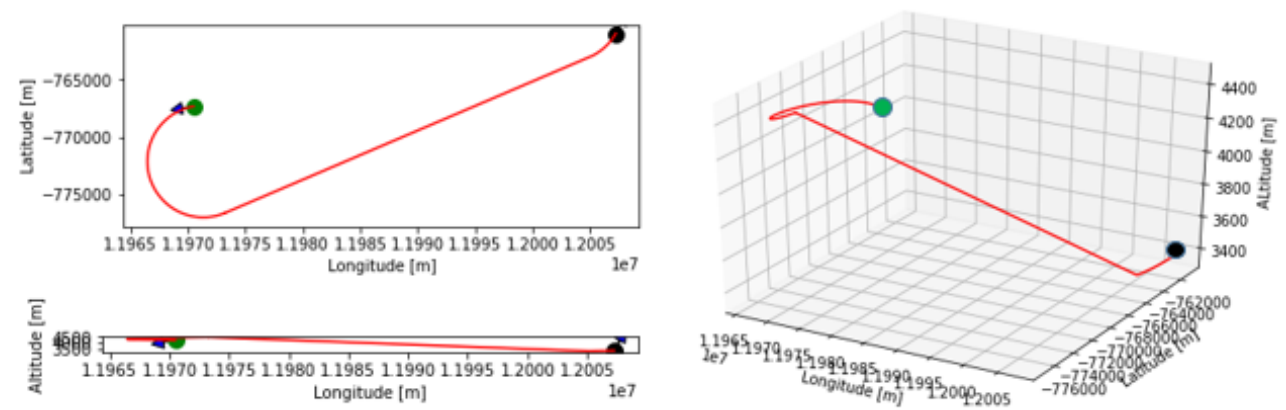

Figure 10. B738 Dubins Airplane Path in Second Closest Waypoint Condition 
In the second closest waypoint condition, the initial aircraft position was close to the DAGOH arrival waypoint. However, the DAGOH arrival waypoint did not fulfill the Dubins Path requirement. The Dubins Airplane Path generated new flight trajectory by using the SUMED arrival waypoint as the new final configuration. In Figure 10 , the flight trajectory had chosen the Dubins Airplane Path Left-Straight-Left (LSL) as the optimal combination path. The aircraft also needed to descent to the final configuration because the aircraft altitude was higher than the final waypoint altitude.

\subsection{Different Bank Angle and Flight Path Angle Values}

In this section, Dubins Airplane Path was simulated in three conditions using B738. First, the simulation was conducted on the maximum bank angle $25^{\circ}$ and the flight path angle $45^{\circ}$.
Second, the simulation was conducted on the maximum bank angle $20^{\circ}$ and the flight path angle $30^{\circ}$. Last, the simulation was conducted on the maximum bank angle $15^{\circ}$ and the flight path angle $15^{\circ}$. The purpose of this simulation is to understand what the effect of bank angle and flight-path angle at this Dubins Airplane Path Simulation.

The simulation performed on the left side of departure route. The aircraft position started at the 108.332 East longitude, -6.715 South latitude, 2127.030-meter altitude and $340^{\circ}$ orientation angle. After Dubins Path calculated the initial waypoint, the flight trajectory generation obtained the GAPIT arrival waypoint as the shortest distance final waypoint (Figure 11). The GAPIT arrival waypoint had a position in the 108.560 East longitude, -6.698 South latitude, 4419.6-meter altitude, and $149^{\circ}$ orientation angle.

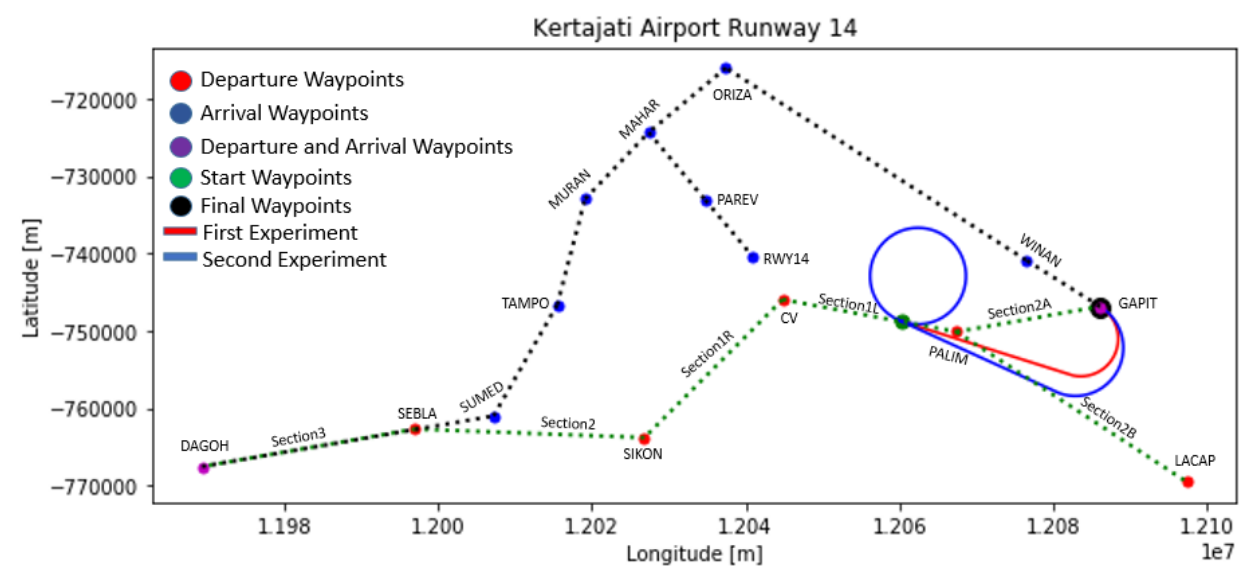

Figure 11. B738 Flight Trajectory in Different Bank Angle and Flight Path Angle Values on the Kertajati Airport
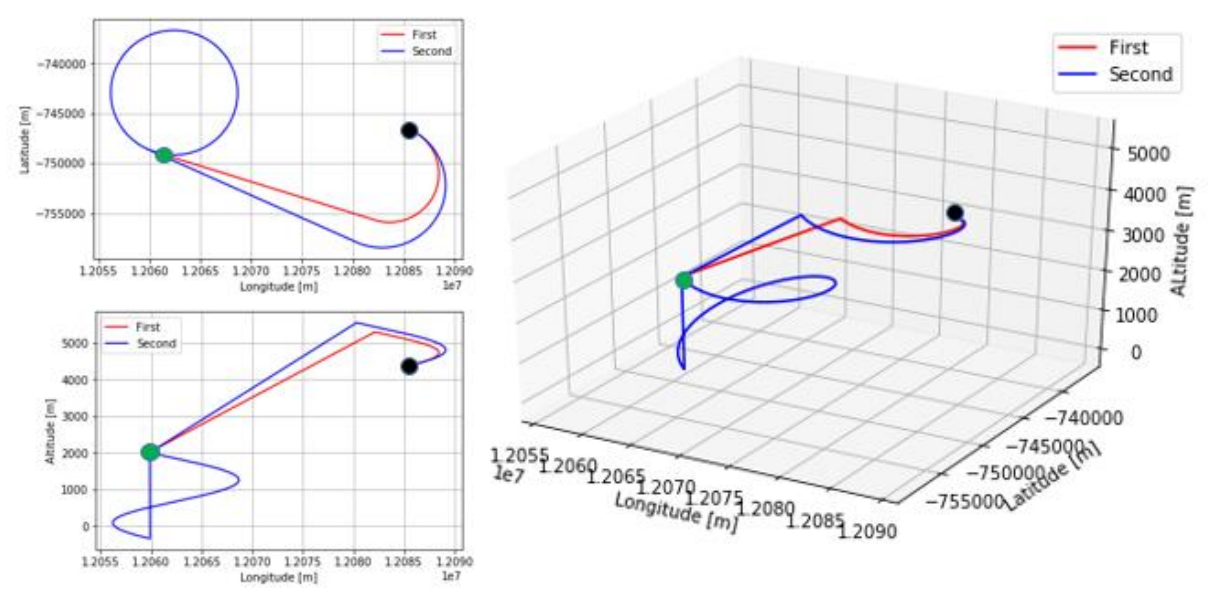
Figure 12: B738 Dubins Airplane Path in Different Bank Angle and Flight Path Angle Values

In this condition, the first simulation was successful to operate a normal flight trajectory with correct Dubins Airplane Path. For the second simulation, the flight trajectory was also successfully created. However, it cannot be implemented to the aircraft. The Figure 12 showed that the flight trajectory caused the aircraft to descent while turning from the initial position and suddenly the aircraft went up vertically to the initial position. This phenomenon is impossible for the aircraft because the aircraft does not fly vertically. The phenomenon also can happen because the flight-path angle is too small for the B738 aircraft to go from the aircraft initial position to the final position.

The results also shown the aircraft bank angle affected the aircraft turn radius and the position of final center of circle (c). The bigger bank angle produces the smaller turning radius. While, the aircraft flight path angle affected the length of Dubins Path and the gamma $(\gamma)$ value.

\section{CONCLUSIONS}

This work developed a flight trajectory generation for a fixed-wing aircraft to be used in the Return to Base situation. The Dubins Path method was used to generate the emergency flight trajectory from the aircraft RTB position to the closest arrival waypoint. The Dubins Path was assisted by the Vector-Field methodology.

Based on the simulation results, the Dubins Airplane Path success to generate the emergency flight trajectory based on the Kertajati Airport flight procedure. The Dubins Airplane Path also can be operated in different simulation conditions. The Vector-Field methodology succeeds to assist the Dubins Airplane Path to generate the emergency flight trajectory. The Dubins path results show that the increase in aircraft turn radius is due to an increase in aircraft velocity and a decrease in aircraft bank angle. While, the increase in Dubins Path line is caused by a decrease in the aircraft flight path angle.

According to the finding in this flight trajectory generation, there are various recommendations needs to be included in the future developments. First, the Dubins Airplane Path simulation should consider another environmental problem (Windy weather or Storm weather). Second, the RTB situation should consider the technical problem factor (Engine Failure, Bird Strike). Third, the Dubins Airplane Path also should consider different case of altitude differences (Medium and High). Also, the future work needs to perform a validation of the resulted Dubins Airplane Path.

\section{REFERENCES}

1. L. E. Dubins, On Curves of Minimal Length with a Constraint on Average Curvature, and with Prescribed Initialand Terminal Positions and Tangents, Am. J. Math., vol. 79, no. 3, pp. 497-516, 1957.

2. S. Hota and D. Ghose, Optimal Path Planning for an Aerial Vehicle in 3D Space, IEEE Conf. Decis. Control, pp. 4902-4907, 2010.

3. X. Yu and J. Y. Hung, A Genetic Algorithm for the Dubins Traveling Salesman Problem, Inst. Electr. Electron. Eng., pp. 1256-1261, 2012.

4. Y. Lin and S. Saripalli, Path planning using 3D Dubins Curve for Unmanned Aerial Vehicles, Inst. Electr. Electron. Eng., no. October 2016.

5. T. Mclain, R. W. Beard, and M. Owen, Implementing Dubins Airplane Paths on Fixed- wing UAVs, in Handbook of Unmanned Aerial Vehicles, 2014, pp. 16771701.

6. I. Lugo-cárdenas, G. Flores, S. Salazar, and R. Lozano, Dubins 
path generation for a fixed wing UAV, Int. Conf. Unmanned Aircr. Syst., no. May 2014, pp. 339-346, 2014.

7. S. Paul, F. Hole, A. Zytek, and C. A. Varela, Flight Trajectory Planning for Fixed-Wing Aircraft in Loss of Thrust Emergencies. Second Int. Conf. InfoSymbiotics, pp. 1-32, 2017.

8. A. Giese, A Comprehensive, Stepby-Step Tutorial to Computing Dubin's Paths, Andy G's Blog, 2012.

9. A. M. Shkel and V. Lumelsky, Classification of the Dubins Set, Rob. Auton. Syst., vol. 34, no. 4, pp. 179-202, 2001.

10. D. G. C. A. of Indonesia, WICA AD 2-1SD AD 2 - 9. AMDT 70. AIM Indonesia, 2018. 